Markus Pfisterer, Isabella Mayer

\section{ÜBERSICHTEN/ REVIEWS}

In der klinischen Praxis steht der Arzt oft Patienten gegenüber, die über monateoder jahrelang andauernde chronische Beschwerden wie Kopfschmerzen, MagenDarm-Probleme, hohen oder niedrigen Blutdruck, Hautausschläge usw. klagen. Die Symptome ähneln einer Allergie, Allergietests sind jedoch negativ. Diese Patienten haben oft jahrelange Odysseen durch Arztpraxen und Spezialkliniken hinter sich. Therapieansätze werden mangels Erfolg oft abgebrochen. Da die Ursache der Symptome unerkannt bleibt, werden die Beschwerden oft als psychisch bedingt eingestuft.

Beim Auftreten von derartigen unklaren allergieähnlichen Symptomen muss der behandelnde Arzt an die Möglichkeit einer Histaminintoleranz (HIT), genauer gesagt an eine biogene Aminintoleranz (BAI) als eine der häufigsten Form der Nahrungsmittelunverträglichkeit denken [7]. Das Krankheitsbild der Histaminintoleranz ist schon seit beinahe 20 Jahren beschrieben [11] und trotz allem besteht noch immer Aufklärungsbedarf in vielen Bereichen.

Histaminintoleranz tritt auf, wenn der Körper mit der Nahrung aufgenommenes Histamin nicht rasch genug im Darm abbauen kann. Für den Abbau von Histamin und anderen biogenen Aminen ist das Enzym Diaminooxidase (DAO; EC 1.4.3.6) an der Darmwand verantwortlich [1, 2]. Dabei spielt die absolute Menge von biogenen Aminen nur eine untergeordnete Rolle, entscheidend ist das Verhältnis zwischen verfügbarer Enzymaktivität und der Menge von Aminen.

\title{
Histaminintoleranz - aktueller Stand der Technik von Diagnose und Therapie
}

\section{Zusammenfassung}

Histaminintoleranz zeichnet sich durch eine Vielfalt von oft diffusen, schlecht zuzuordnenden Symptomen aus, welche mit der klassischen klinischen Diagnostik nicht erfasst werden können. Die Autoren stellen ein neues, dreistufiges Konzept zur Diagnose und Therapieunterstützung dieses Krankheitsbildes vor. Die Schritte umfassen einen standardisierten Anamnese-Fragebogen, einen klinisch-chemischen Nachweis der Diaminooxidaseaktivität und ein diätetisches Lebensmittel, das die Enzymdefizienz ausgleicht.

\section{Schlüsselwörter}

Histaminontoleranz, Histaminose, Nahrungsmittelunverträglichkeit, Pseudoallergie, Diaminooxidase, Histamin.

\section{Abstract}

Histamine intolerance is characterized by a large variety of diffuse symptoms, which can not be well attributed, and which can not be grasped by classical clinical diagnostics. The authors present a new, three-phase concept for the diagnosis and therapeutical support of this clinical picture. The steps include a standardized anamnestic questionnaire, clinical and chemical evidence of the diamine oxidase activity, and dietetic food, which balances the enzyme deficiency.

\section{Keywords}

Histamine intolerance, histaminosis, food intolerance, pseudoallergic reaction, diamine oxidase, histamine.

Die Prävalenz für eine klinisch manifeste Histaminintoleranz liegt bei etwa $1 \%$ der Bevölkerung [6, 7], allerdings leiden bis zu $20 \%$ der Bevölkerung mehr oder weniger stark unter fallweiser Nahrungsmittelunverträglichkeit, die in den meisten Fällen auch auf eine Histaminintoleranz zurückzuführen ist.

\section{Biochemische Aspekte}

Histamin [2-(4-Imidazolyl)-ethylamin] gehört in die Substanzgruppe der biogenen Amine, die ubiquitär in der Natur vorkom- men und häufig bei Verderbnis- und Fäulnisprozessen gebildet werden. Insbesondere Histamin ist im menschlichen Organismus von großer Bedeutung und mittlerweile sehr gut untersucht. Die blutdrucksenkende Wirkung von exogenem Histamin wurde erstmals 1910 beschrieben, das Vorkommen in Geweben (Histos) ist seit 1927 dokumentiert. 1932 wurde die Funktion als Mediator allergischer Reaktionen geklärt.

Histamin entsteht aus der Aminosäure Histidin durch oxidative Decarboxylierung durch die L-Histidin-Decarboxylase(Abb.1). 


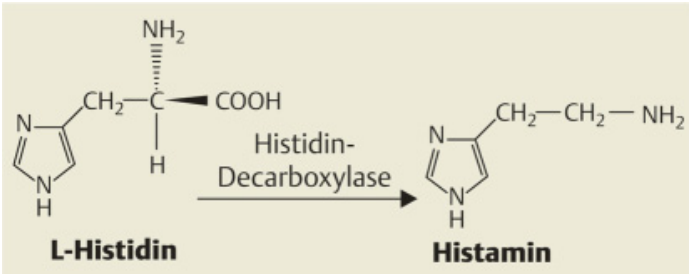

Abb. 1: Bereitstellung von Histamin aus Histidin.

Als lebensnotwendiger Botenstoff wird es u.a. in Mastzellen, basophilen Leukozyten und Thrombozyten produziert und gespeichert. Weiter ist Histamin ein essenzieller Neurotransmitter und somit auch im Nervensystem vorhanden. Neuronales Histamin ist für die nun folgenden Ausführungen jedoch nicht relevant. Bei Bedarf oder externen Stimuli wird Histamin vor allem aus den Mastzellen freigesetzt (Degranulation) und löst damit eine Vielzahl von Reaktionen aus, auf die hier nicht näher eingegangen werden soll. Die Degranulation der Mastzellen erfolgt typischerweise IgE-vermittelt, was der allergischen Sofortreaktion vom Typ 1 entspricht. Es kann jedoch durch bestimmte Histaminliberatoren auch zu einer nicht IgE-vermittelten Degranulation mit allergieähnlichen Symptomen kommen. Diese Reaktion wird als Pseudoallergie bezeichnet. Eine Auswahl typischer Histaminliberatoren zeigt Tabelle 1.

Histamin wird ausschließlich von zwei Enzymen abgebaut (Abb. 2):

- Diaminooxidase (DAO; EC 1.4.3.6) führt zu einer oxidativen Deaminierung (extrazellulär); im Gastrointestinaltrakt relevant,

- Histamin-N-Methyl-Transferase methylierung (intrazellulär). Aktivität reduziert sein: ten), min $\mathrm{B}_{6}$ (selten), gien), etc. [9].

\section{Physiologische Aspekte}

(HNMT; EC 2.1.1.8) führt zu einer Ring-

Die für den Abbau von oral aufgenommenem Histamin verantwortliche DAO kann durch eine Vielzahl von Faktoren in ihrer

- genetisch bedingte zu geringe Enzymsynthese oder -aktivität (äußerst sel-

- Mangel an Kofaktoren z. B. Kupfer, Vita-

- entzündungsbedingte reduzierte Enzymproduktion in der Darmschleimhaut [10] (chronische Entzündungen oder intestinale Nahrungsmittelaller-

- Hemmung der Enzymaktivität durch z.B. Acetaldehyd, dem Abbauprodukt von Alkohol oder Inhaltsstoffen bestimmter Medikamente [5] (Tab. 2),

- hoher Überschuss an weiteren biogenen Aminen wie Putrescin, Cadaverin

Wird Histamin aufgrund eines oder mehrerer der oben genannter Faktoren nach der Zufuhr in den Darm nicht recht-

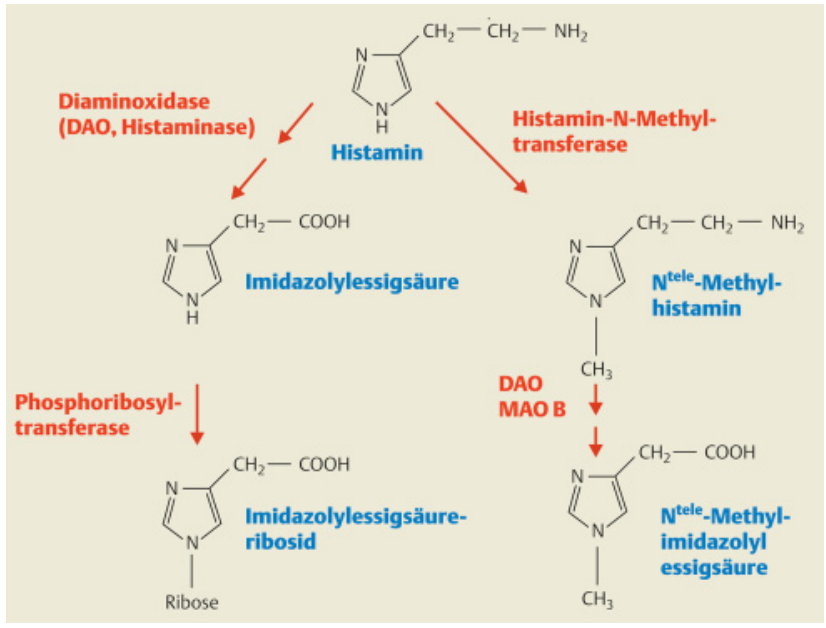

Abb. 2: Abbau von Histamin.
Tab. 1: Histaminliberatoren (modifiziert nach [6]).

- Erdbeeren
- Zitrusfrüchte
- Tomaten
- Meeresfrüchte
- salicylathaltige Nahrungsmittel
fraglich:
- Ananas
- Kiwi
- Milch

Tab. 2: DAO-hemmende Substanzen.

- Alkohol (und dessen Abbauprodukt
Acetaldehyd)
- viele häufig verordnete Arzneimittel
(Auswahl):
- Acetylzystein
- Ambroxol
- Diclofenac
- Azetylsalizylsäure
- Ibuprofen
- Metoclopramid
- Metamizol
- Theophyllin

- Verapamil

zeitig und effizient abgebaut, kann es nach dem Durchtritt durch die Darmwand in nahezu allen Körperzellen Reaktionen auslösen [11]. Daher sind die dadurch getriggerten Beschwerden entsprechend vielfältig und können immer wieder anders sein („Chamäleon“). Es sind vier verschiedene Histaminrezeptoren mit jeweils leicht unterschiedlichen Wirkungen bekannt (H1 - H4). Dies erklärt die Vielfalt der Symptome einer endo- oder exogenen Histamindysbalance.

Grundsätzlich kann zwischen „zentralnervösen“ und „gastrointestinalen“ Beschwerden unterschieden werden (Tab. 3).

Oft, aber charakteristischerweise nicht immer, reagieren Betroffene mit den gleichen Beschwerden. Diese treten meist unmittelbar bis ca. zwei Stunden nach dem Essen auf und können unter Umständen bis zu einem halben Tag oder länger andauern.

\section{Diagnose}

Bevor eine Histaminintoleranz, wie weiter unten beschrieben ist, diagnostiziert werden kann, muss eine über das Im- 
Tab. 3: Gastrointestinale und zentralnervöse Beschwerden.

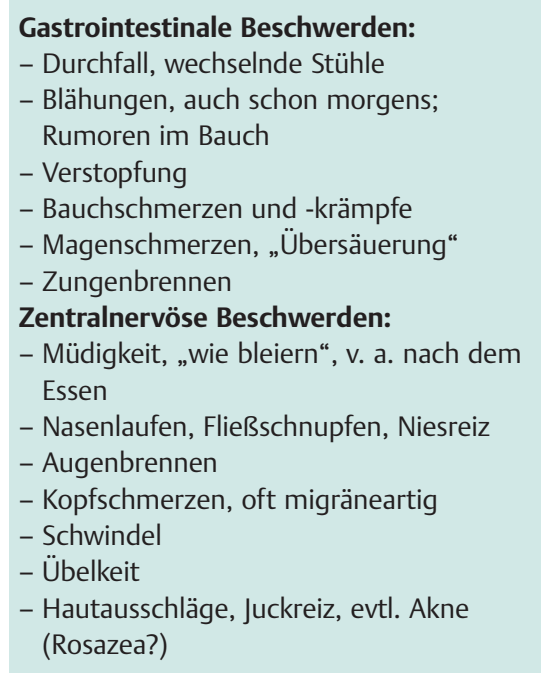

munsystem modulierte Reaktion (IgE, IgG, IgG4, LTT-Typ IV) ausgeschlossen werden [3]. Anschließend muss mit dem Patienten im Rahmen eines ausführlichen Gespräches das Auftreten der Beschwerden im Zusammenhang mit der Ernährung erarbeitet werden. Sowohl die Intensität als auch die Häufigkeit und Dauer der Symptome muss in einem Fragebogen (Tab. 4) erfasst und gewichtet werden. Ein derartiger Fragebo-

Tab. 4: Prinzip des Fragebogens.

1) Dokumentation und Gewichtung der
Leitsymptome
- Durchfall
- Blähungen
- Darmkrämpfe
- Bauchschmerzen
- Kopfschmerzen
- Hautausschläge

2) Feststellen von bekannten Intoleranzen gegen Nahrungsmittel

- Sekt

- Rotwein

- andere alkoholische Getränke

- Fisch

- reifer Käse

- fermentierte Würste

- Tomaten

- Zitrusfrüchte

3) Die gewichteten Punkte werden addiert und ergeben somit die patientenspezifischen HIT-Punkte. Da häufig parallel weitere Nahrungsmittelintoleranzen vorkommen (Fruktose-, Laktoseintoleranz, Glutensensitivität), wurde für die zusätzlichen Diagnosen ein Korrekturfaktor eingeführt, der die ermittelten Punkte entsprechend reduziert.

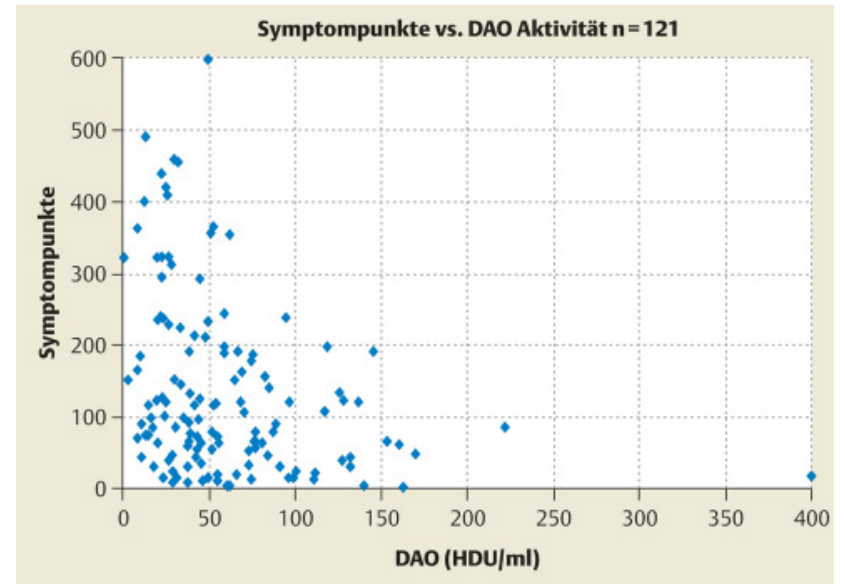

Abb. 3: Symptomscore und DAO-Aktivität.

gen wird von den Autoren derzeit entwickelt und evaluiert.

Im nächsten Schritt wird die Aktivität der DAO im Serum oder Plasma bestimmt. Diese korreliert bekanntermaßen sehr gut mit der Aktivität in den Enterozyten [13].

Zurzeit sind zwei unterschiedliche Testsysteme verfügbar, um die DAO-Aktivität zu messen. Die bisher üblichen Testsysteme zur Bestimmung der Aktivität der Diaminooxidase verwenden radioaktiv markiertes Putrescin als Substrat, dessen Abbau mit der Abbaukapazität von Histamin korreliert. Dieser REA-Test ist inzwischen gut etabliert und gilt als Standard [8]. Leider können nur Labore mit Zulassung für radiologische Testmethoden den Test ausführen. Inzwischen wurde jedoch auch eine auf einem Enzyme-linked-Immunoassay basierende Testmethode entwickelt, die sehr viel einfacher zu handhaben ist und weniger Beschränkungen und Auflagen unterliegt (D-HIT $\left.{ }^{\circledR}\right)$. Bei diesem Enzymassay dient nicht Putrescin als Substrat, sondern es wird direkt die Abbaufähigkeit des Serums oder Plasmas für eine definierte Menge Histamin gemessen. Da die Reaktionsgeschwindigkeit des Enzyms mit Putrescin und Histamin unterschiedlich ist [12], repräsentiert die Histamin-basierte Testversion die direkte Korrelation zur Histaminintoleranz. Für den Test sind $50 \mu \mathrm{l}$ Serum oder Plasma nötig, die aufgrund der guten Stabilität des Enzyms auch für wenige Tage bei $4^{\circ} \mathrm{C}$ gelagert werden können. Die Aktivität der DAO wird in Histamin Degrading Units (HDU) pro ml angegeben, wobei 1 HDU jene Aktivität an- gibt, bei der DAO unter Assaybedingungen $1 \mathrm{pmol} / \mathrm{ml}$ Histamin abbaut.

Die mit dem Fragebogen ermittelten HIT-Punkte zeigen eine gute inverse Korrelation zur gemessenen Aktivität der DAO im Blut. Abbildung 3 zeigt auch, dass eine niedrige Aktivität der DAO nicht notwendigerweise eine starke Symptomatik mit sich bringen muss. Um die Dysbalance von Histamin und DAO-Aktivität festzustellen, ist daher auch die Ermittlung des Histaminspiegels als ergänzender Laborparameter zu empfehlen. Aus der Zusammenstellung von Anamnese, Klinik, DAO-Aktivität und Histaminwert kann so die Diagnose der Histaminintoleranz zuverlässig gestellt werden.

\section{Therapie}

Der primäre Ansatz einer HIT-Therapie ist eine histaminarme Diät. Tabellen mit entsprechenden Nahrungshinweisen sind zur Genüge verfügbar [14]. Ergänzend sei darauf hingewiesen, dass Histamin hitzeund kältestabil ist und durch küchentechnische Methoden wie Einfrieren, Kochen, Backen oder Mikrowellenerhitzung nicht zerstört werden kann.

Parallel und ergänzend ist eine Kontrolle und evtl. Sanierung der Darmflora indiziert. Störungen der Darmflora, z.B. durch Überwucherung mit Gärungs- und Fäulniskeimen oder Hefepilzen, wie sie häufig nach wiederholten Antibiotikatherapien auftreten, führen zur Funktionseinschränkung der Darmzellen sowie zu einer leichten entzündlichen Veränderung der 


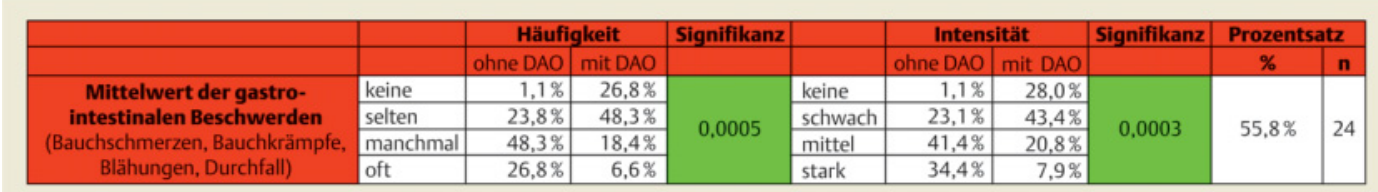

Gastrointestinale Beschwerden
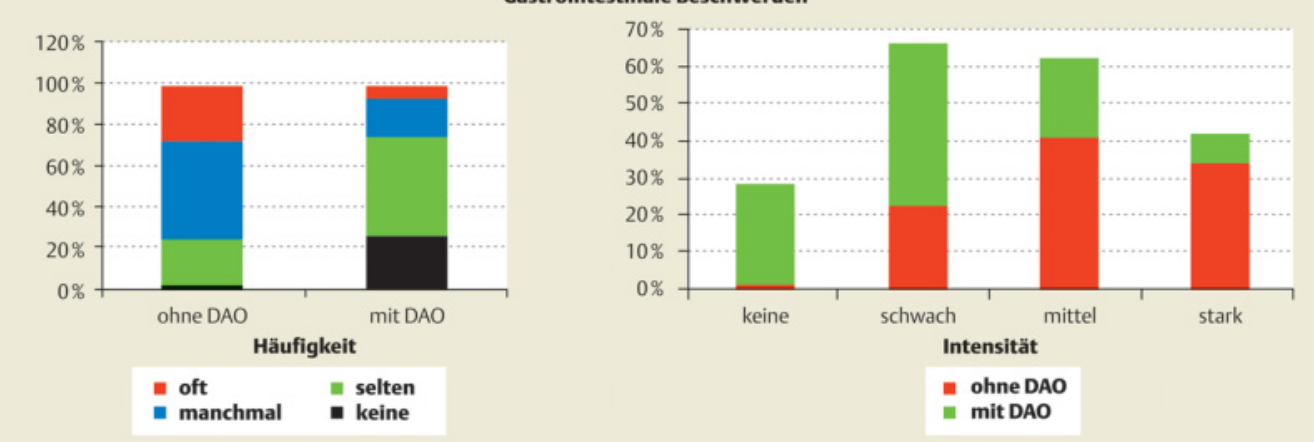

Abb. 4: Verbesserung der Symptomatik durch Enzymsubstitution.

Darmwand und einer vermehrten Durchlässigkeit derselben (Leaky-Gut-Syndrome). Die Sanierung der Darmflora erfolgt mit desinfizierenden oder pilztötenden Substanzen sowie mit Prä- und Probiotika und Bakterienlysaten und sollte von einem erfahrenen Arzt durchgeführt werden. Unterstützend kann auch eine Darmreinigung durch eine Fastenkur oder eine Colon-Hydro-Therapie durchgeführt werden.

Die HIT bzw. BAI tritt selten als alleiniges Krankheitsbild auf. Häufig finden sich weitere Nahrungsmittelintoleranzen wie Zuckerintoleranzen (Fruchtzucker, Milchzucker, Sorbit). Diese Stoffwechselstörun- gen müssen bei der Diätplanung unbedingt ebenfalls berücksichtigt werden.

Mikronährstoffe (Vitamine, Mineralstoffe, Aminosäuren, Fettsäuren) sind wichtige Helfer unserer Körperfunktionen. Jegliches Defizit führt zwangsläufig zu einer Funktionseinbuße. Dies kann im Falle der BAI dazu führen, dass entweder das histaminabbauende Enzym Diaminooxidase (DAO) zu langsam arbeitet, zu viel Histamin aus den Körperspeichern freigesetzt wird (Mastzelldegranulation) oder eine Kombination aus beiden Faktoren vorliegt. Daher werden durch geeignete Untersuchungsverfahren die Defizite aufgedeckt und indi-

Tab. 5: Orthomolekulare Empfehlungen bei Histamindysbalancen.

\begin{tabular}{|c|c|c|}
\hline Vitalstoff & Funktion & Dosierung \\
\hline Vitamin C & $\begin{array}{l}\text { beteiligt am Histaminabbau; nied- } \\
\text { rige Vitamin-C-Spiegel korrelieren } \\
\text { mit hohen Histaminspiegeln [4] }\end{array}$ & $\begin{array}{l}\text { oral: } 1-3 \mathrm{~g} \\
\text { parenteral: bis } 20 \mathrm{~g}\end{array}$ \\
\hline Vitamin $\mathrm{B}_{6}$ & $\begin{array}{l}\text { beteiligt am Histaminabbau; } \\
\text { Decarboxylierung ist Pyridoxal- } \\
\text { phosphat-abhängig }\end{array}$ & oral: $10-100 \mathrm{mg}$ \\
\hline Kalzium & $\begin{array}{l}\text { Stabilisierung der Mastzell- } \\
\text { membran }\end{array}$ & $\begin{array}{l}\text { oral: } 1000-1500 \mathrm{mg} \\
\text { parenteral: } 10 \mathrm{ml} \mathrm{Ca} 10 \% \\
\text { (entspr. } 2,25 \mathrm{mmol} \mathrm{Ca}^{2+} \text { ) }\end{array}$ \\
\hline Methionin & $\begin{array}{l}\text { Methylgruppendonator: Abbau des } \\
\text { Histamins zu N-Methylhistamin } \\
\text { (v. a. bei ZNS-Symptomen) }\end{array}$ & $\begin{array}{l}500-1500 \text { mg (Cave: zur Verbeu- } \\
\text { gung von Hyperhomozysteinämie } \\
B_{6}, B_{12} \text { und Folsäure geben) }\end{array}$ \\
\hline Zink & $\begin{array}{l}\text { wichtig für die Aktivierung von } \\
\text { Vitamin } B_{6}\end{array}$ & oral: 10-25 mg \\
\hline Diaminooxidase & $\begin{array}{l}\text { baut Histamin im Darm ab, bevor } \\
\text { dieses resorbiert werden kann }\end{array}$ & $\begin{array}{l}\text { oral: 1-3 Kapseln ( } 1 \text { Kapsel enthält } \\
\text { ca. } 10000 \text { HDU Enzymaktivität) }\end{array}$ \\
\hline
\end{tabular}

viduell substituiert (Tab. 5). Besonders wichtig sind dabei die Nährstoffe Vitamin C, Kupfer, Magnesium, Kalzium, Zink, Omega-3-Fettsäuren, Linol- und Linolensäuren (aus Schwarzkümmelöl).

Die ergänzende Substitution des Enzyms Diaminooxidase als diätetisches Lebensmittel führt unmittelbar zu einer signifikanten Reduktion der Symptome und verbessert die Lebensqualität der Betroffenen sehr stark. Das Enzym baut direkt im Darm Histamin aus den Lebensmitteln ab und reduziert so die Histaminbelastung des Organismus. Es ist besonders geeignet z.B. zur Bestätigung des Verdachts einer BAI sowie am Anfang der diätetischen Maßnahmen, solange sich der Betroffene noch nicht genau mit den Lebensmitteln auskennt. Im weiteren Verlauf nimmt man die Kapseln bei Bedarf, z. B. unterwegs, im Urlaub oder bei anderweitigem auswärtigem Essen (Restaurant, Party), dessen Histamingehalt man nicht genau abschätzen kann. Eine erste Beobachtungsstudie zeigt die rasche und signifikante Verbesserung der Symptomatik durch die Enzymsubstitution (Abb. 4).

\section{Diskussion}

Wir haben in der Praxis in den vergangenen drei Jahren bei über 350 Patienten die Aktivität der Diaminooxidase ermittelt. Bei 160 Patienten lag die Aktivität sicher im Bereich einer starken Unterfunktion, bei 
weiteren 80 Patienten im Bereich einer leichten Unterfunktion. Dies zeigt die enorme praktische Relevanz, die die Histaminintoleranz hat. Mit einer gezielten Anamnese, einem standardisierten Fragebogen sowie einer sinnvollen Labordiagnostik (DAO, Vitamin $\mathrm{B}_{6}$, Kupfer, Vitamin C, evtl. Histamin) kann die Diagnose sicher gestellt werden und dann den oftmals verzweifelten Patienten eine wirksame, nebenwirkungsfreie und kostengünstige Therapie angeboten werden.

Nach erfolgreicher Diagnose und Einleitung therapeutischer Maßnahmen bessern sich die Beschwerden oft dramatisch und sehr rasch. Nach etwa zwei Wochen wird häufig eine sehr gute Beschwerdelinderung erreicht, die auf Dauer anhaltend ist. Allerdings konnten wir beobachten, dass es bei einigen Patienten mit Einleitung der Therapie zunächst zu einer akuten Erstverschlimmerung (oder vielleicht sogar zu Entzugssymptomen) durch die Histaminreduktion kommen kann. Auch diese Phase der Behandlung ist zeitlich begrenzt und dauert meist nicht länger als drei Tage, in seltenen Fällen bis zu einer Woche an. In dieser Phase ist eine enge Führung des Betroffenen durch einen erfahrenen Therapeuten wichtig.

Häufig kommt es zu einer dauerhaften Besserung sowie zu einer Normalisierung der Enzymaktivität, wodurch sich auch die Verträglichkeit der Lebensmittel wieder deutlich bessert bis hin zu einer normalen Lebensmitteltoleranz. Die Zeiträume hierfür sind allerdings sehr unterschiedlich und können bis zu zwei Jahren dauern.

\section{SERVICEKASTEN}

Labors, die Diaminooxidase bestim-

men, erfahren Sie bei:

Sciotec Diagnostic Technologies $\mathrm{GmbH}$

Simmeringer Hauptstraße 24

1110 Wien

Österreich

E-Mail: info@sciotec.com

Präparate, die zur Therapieunterstützung bei Histaminintoleranz geeignet sind (Auswahl):

Daosin $^{\circledR}$ Kapseln (Melbrosin):

tgl. 1-2 Kps. zur Mahlzeit

Vitamin $B_{6}$ (z. B. Hevert, Pascoe): tgl. 25-50 mg morgens

Zink (z. B. Unizink ${ }^{\circledR}$, Zinkotase ${ }^{\circledR}$ ): tgl. 15-50 mg

Vitamin C (z. B. Vitamin C Pascoe, Citrovit $^{\circledR}$ Centropa): tgl. 50-2000 mg

Kupfer (z. B. Kupfer-POS ${ }^{\oplus}$ ): tgl. 1-2 mg

\section{Literatur}

[1] Ahrens F, Gabel G, Garz B, Aschenbach JR: Release and permeation of histamine are affected by diamine oxidase in the pig large intestine. Inflamm Res. 2002; 51 (Suppl 1): S83-S84.

[2] Aschenbach JR, Schwelberger HG, Ahrens F, Fürll B, Gäbel G: Histamine inactivation in the colon of pigs in relationship to abundance of catabolic enzymes. Scand J Gastroenterol. 2006; 41: 712-719.

[3] Bruijnzeel-Koomen C, Ortolani C, Aas K, Bindslev-Jensen C, Bjorksten B, MoneretVautrin D, Wuthrich B: Adverse reactions to food. European Academy of Allergology and Clinical Immunology Subcommittee Allergy. 1995; 50: 623-635.

[4] Clementson CAB: Histamine and Ascorbic Acid in Human Blood. J Nutr. 1980; 110: 662-668.

[5] Hui JY, Taylor SL: Inhibition of in vivo histamine metabolism in rats by foodborne and pharmacologic inhibitors of diamine oxidase, histamine $\mathrm{N}$-methyltransferase, and monoamine oxidase. Toxicol Appl Pharmacol. 1985; 81: 241-249.

[6] Jarisch R, Götz M, Hemmer W, Missbichler A, Raithel M, Wantke F: Histamin-Intoleranz, Histamin und Seekrankheit. 2. Aufl. Stuttgart: Thieme Verlag; 2004.
[7] Maintz L, Bieber T, Novak N: Die verschiedenen Gesichter der Histamin-Intoleranz. Deutsches Ärzteblatt. 2006; 103 : 3477-3483.

[8] Mayer I, Missbichler A, Wantke F, Focke M, Reichl H, Winter M, Jarisch R: Optimized radioextraction assay for quantitative determination of diamine oxidase (DAO) activity in human serum and blood. Allergologie. 2005; 28: 1-8.

[9] Nilsson BO, Kockum I, Rosengren E: Inhibition of diamine oxidase promotes uptake of putrescine from rat small intestine. Inflamm Res. 1996; 45: 513-518.

[10] Raithel M, Ulrich P, Hochberger J, Hahn EG: Measurement of gut diamine oxidase activity. Diamine oxidase as a new biologic marker of colorectal proliferation? Ann N Y Acad Sci. 1998; 859: 262-266.

[11] Sattler J, Hafner D, Klotter HJ, Lorenz W, Wagner PK: Food-induced histaminosis as an epidemiological problem: plasma histamine elevation and haemodynamic alterations after oral histamine administration and blockade of diamine oxidase (DAO). Agents Actions. 1988; 23: 361-365.

[12] Schwelberger HG, Bodner E: Purification and characterization of diamine oxidase from porcine kidney and intestine. Biochim Biophys Acta. 1997; 1340: 152-164.

[13] Tufvesson G, Tryding N: Determination of diamine oxidase activity in normal human blood serum. Scand J Clin Lab Invest. 1969; 24: $163-168$

[14] Wantke F, Gotz M, Jarisch R: Histamine-free diet: treatment of choice for histamineinduced food intolerance and supporting treatment for chronic headaches. Clin Exp Allergy. 1993; 23: 982-985.

\section{Korrespondenzadresse}

Dr. med. Markus Pfisterer Nordstraße 28, 74076 Heilbronn E-Mail: info@DrPfisterer.de

Dr. Isabella Mayer Institut für Biochemie und molekulare Zellbiologie, Universität Wien Dr. Bohrgasse 9, 1030 Wien Österreich 